\title{
Sensor data collecting system for railway facilities
}

\author{
Y. Watanabe, N. Sato \& R. Tsuchiya \\ Railway Technical Research Institute, Facilities Management Systems, \\ Kokubunji-shi, Tokyo, Japan
}

\begin{abstract}
The application of health monitoring systems to railway structures is now being discussed and some experimental systems have already been implemented and tested. It is expected that health monitoring systems will bring positive effects on structural maintenance. We have developed a health monitoring system in which one can select two methods of data collection: one is a data collecting system with a RFID tag and a PDA (Personal Digital Assistant) and another is a data collecting system with a wireless radio network (ZigBee) and a cellular phone network. This paper describes the overview of our sensor data collecting system and its application to railway structures.
\end{abstract}

Keywords: wireless sensor network, structural health monitoring.

\section{Introduction}

Railway facility maintenance is essential to ensure safe train operation. Maintenance workers usually inspect facilities by visual check, which is a laborintensive and time-consuming process. Their decision on the soundness of each facility is mainly based on the heuristics acquired through their long experiences and we are facing a lack of experienced inspectors. Therefore, we need to establish a new framework in which maintenance workers can inspect facilities in a more efficient and less labor-intensive manner.

We have developed a sensor data collecting system for health monitoring of railway structures such as viaducts and tunnels. In this system, we can select an appropriate way to collect data depending on deformation of the structure: by RFID tag or low-power radio. Data collected by low-power radio are stored in a relay station, and are transmitted to the maintenance office using the mobile phone network. 
The most important issue that we have to cope with in connection with radio transmission is the reduction of energy consumption. The system uses low-power radio and we reduced energy consumption by reducing the frequency of sensing and transmitting to the minimal level. In spite of this energy-aware behavior of the system, the data will be transmitted immediately to the maintenance office when a sensor generates a value that exceeds the predefined threshold level. This system deals with damage to RC frames, damage to foundation structures, and cracks in tunnels. The collected data can be easily referred to and analyzed with a PC in the management department. The main functionalities of this system are data reference, data registration, database master maintenance, and data analysis. By using this system, the inspection work of the railway structures can be streamlined and the inspection precision can be improved.

\section{Features of the sensor data collecting system}

The application of a health monitoring system to railway structures is now being discussed and some experimental systems have already been implemented and tested. It is expected that health monitoring systems will bring positive effects for structure maintenance. We have developed a health monitoring system in which one can select two methods of data collection: one is a data collecting system with a RFID tag and a PDA (Personal Digital Assistant) and another is a data collecting system with a wireless radio network (ZigBee) and a cellular phone network.

\subsection{Data collecting system by RFID tag}

The RFID tag adopted in this system is a passive tag. Electronic power is supplied electromagnetically by a tag reader. We use the radio wave of the frequency at $125 \mathrm{kHz}$, which is less vulnerable to the effect of water and metal material. Figure 1 shows the damage detecting sensor. It is made from 3 strain measuring gauge and RFID. The gauge and the tag are connected by a lead wire. Figure 2 shows the RFID tag reader and the PDA. They are connected by

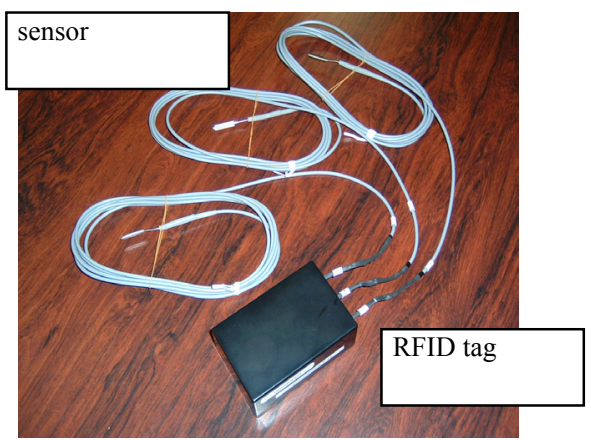

Figure 1: Damage detecting sensor.

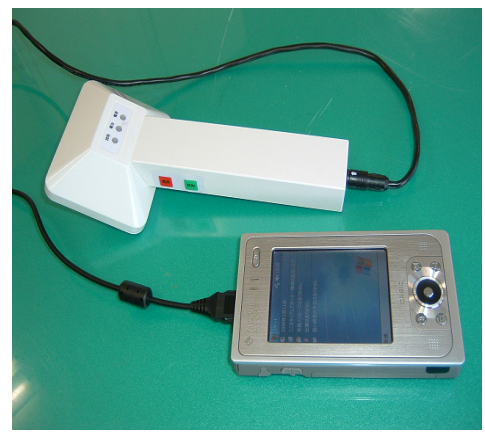

Figure 2: $\quad$ RFID tag reader and PDA. 
RS-232C cable and we can get the data of 3 gauges into the PDA with electric power supplied from the tag reader.

\subsection{Sensor data collecting by radio transmission}

Taking into consideration the locations where sensors will be distributed, it is difficult to charge batteries frequently. Therefore, the energy consumption of sensors must be sufficiently low. Table 1 shows radio devices and specifications that we selected as candidate devices for our health monitoring systems. Figure 3 shows throughput of the data transmission for each device.

Table 1: $\quad$ Specification of low-power radio devices.

\begin{tabular}{|l|l|c|c|c|}
\hline $\begin{array}{l}\text { Radio } \\
\text { device }\end{array}$ & $\begin{array}{l}\text { Frequency } \\
\text { band }\end{array}$ & $\begin{array}{l}\text { Transmission } \\
\text { distance }(\mathrm{m})\end{array}$ & $\begin{array}{c}\text { Transmission } \\
\text { speed }(\mathrm{kbps})\end{array}$ & $\begin{array}{l}\text { Power } \\
(\mathrm{mW})\end{array}$ \\
\hline ZigBee & $2.4 \mathrm{GHz}$ & 30 & 250.0 & 60 \\
\hline $\begin{array}{l}\text { Specified } \\
\text { low-power } \\
\text { radio }\end{array}$ & $429 \mathrm{MHz}$ & $30 \sim 300$ & 2.4 & 59 \\
\hline Weak radio & $315 \mathrm{MHz}$ & $30 \sim 200$ & 2.0 & 66 \\
\hline
\end{tabular}

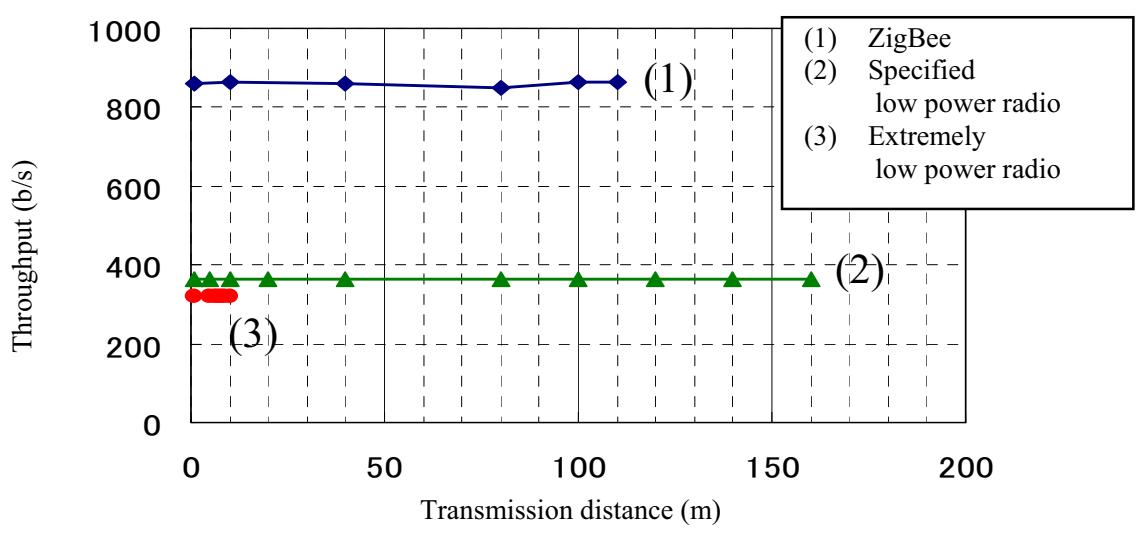

Figure 3: Transmission throughput.

Interference with wireless LANs (IEEE802.11b/g), which are commonly used along railway lines, can be a problem when using wireless technologies in railway fields, but the test result revealed that there is no interference if the "carrier to noise ratio" (C/N ratio) for receiving is greater than $10 \mathrm{db}$. Another method to avoid the interference with wireless LAN is to use a channel different from that used by wireless LAN. After transmitting data, the ZigBee radio device goes into sleep mode, so undesirable effects on wireless LANs can be very small. Since it does not spend so much time on data transmission, data may be 
transmitted quickly while the wireless LAN is inactive. It should also be pointed out that the ZigBee radio device can transmit data in a multi-hop way even if the device cannot transmit data directly to the destination. Taking into consideration such characteristics of radio waves and data transmission, we decided to use the ZigBee radio for data transmission. Figure 4 shows a radio device for the peak sensor. Figure 5 shows a radio device for the $\pi$-shaped displacement measuring sensor. Radio devices periodically get data from sensors by battery power and transmit data to relay stations (Figure 6). Relay stations are operated by solar battery power or $100 \mathrm{~V}$ DC power supply and transmit data to the database server located in the branch office of the railway operator periodically or when data stored in the relay station reaches the predefined amount, by using the cellular phone network.

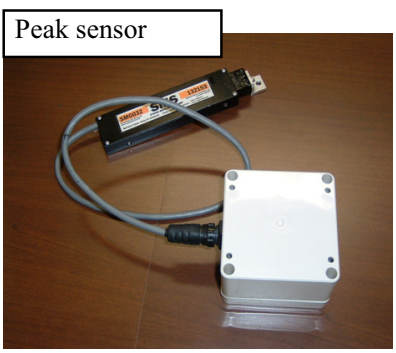

Figure 4: Radio device for the peak sensor.

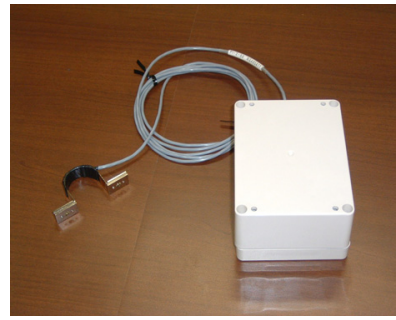

Figure 5: Radio device for the $\pi$-shaped sensor.

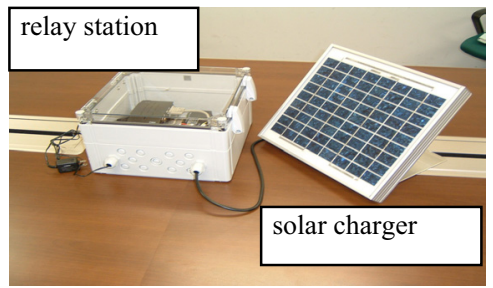

Figure 6: Relay station.

\subsection{Developing an intelligent relay station}

The important issues regarding radio transmission are the electric power supply and the data transmission throughput. In our system, by adopting low-power radio transmission and optimized operation of sensor devices, electric power consumption is greatly reduced. For example, except when relay stations are sensing and/or transmitting data, they fall into sleep mode, thus they consume a very small amount of energy. In addition to this, the volume of transmitted data is reduced by data compression and the removal of duplicated data (i.e. if a data to be sent happened to be the same as the previous one, transmission of it will be 
suppressed). When critical data occurs, the system will immediately transmit the data in spite of the energy saving behavioral characteristics of the relay stations described above. The system also monitors the voltage of the battery, so that the decrease in the residual capacity of the battery will be notified before the functionalities of the relay stations (e.g. data transmission) are totally disrupted.

\subsection{Reference of collected data}

Collected data will be registered on the administration center server, and maintenance officers can refer to the data from a PC at the maintenance office at any time they want. The main programs of this system are data reference, the data register, database master maintenance, and the data analysis program.

(1) Data reference program

By using this system, maintenance officers can refer to collected sensor data and the specifications of structures to which sensors are attached. As is depicted in figure 7, one can refer to the specifications of structures, their pictures, drawings, etc. by using rough drawings or by a conditional search (figure 8).

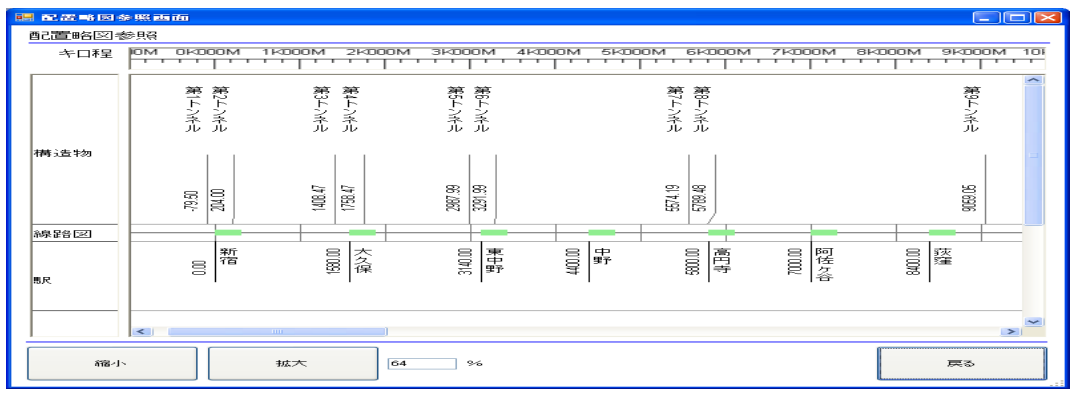

Figure 7: $\quad$ Specifications, pictures and drawings of structures.

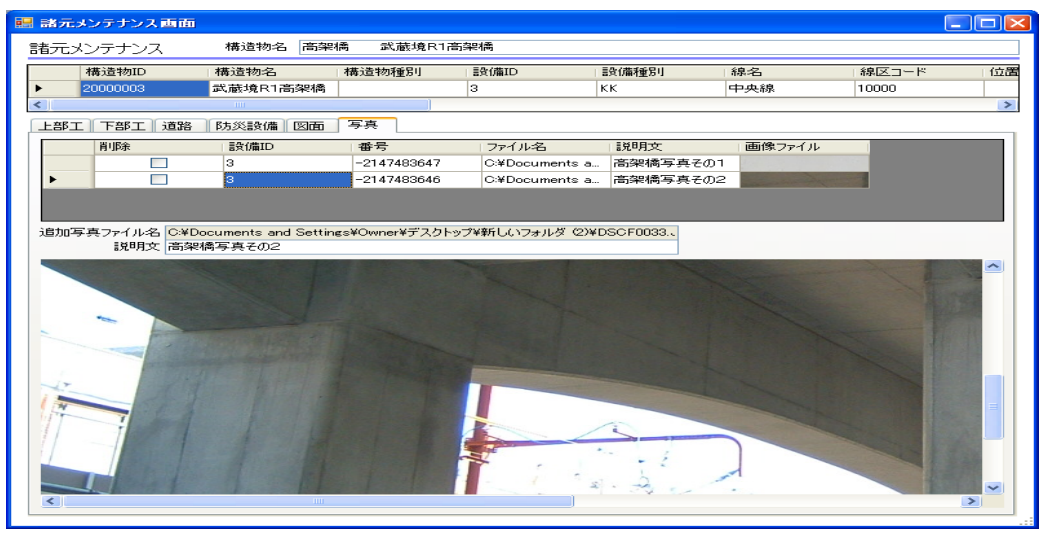

Figure 8: $\quad$ Reference of specifications of structures. 


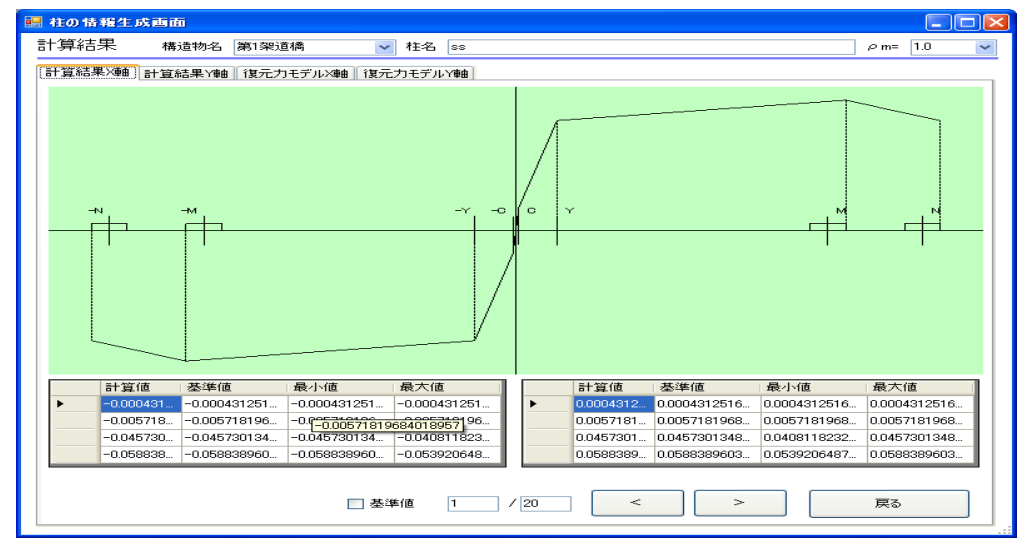

Figure 9: Calculation result based on restoration model.

(2) Data registration program

Data collected by using the RFID tag are registered into the database by connecting the PDA with the client PC. Data collected by using radio transmission are directly registered into the database via the cellular phone network and the network server.

(3) Database master maintenance program

This program updates, adds and deletes master information about specifications of structures, sensor types and measuring contents as well as sensor locations.

(4) Data analysis program

This progam carries out time-series analysis by using measured data registered in the database. Based on the data collected from the sensors, it evaluates the damage level of the RC rigid bridge by using the restoration model (Figure 9), which has already been developed.

\section{Application for civil engineering structures}

In this section, we explain the outline of three types of sensors that we applied to bridges and tunnels .

\subsection{Application for damage level detection for rigid-frame bridges}

RC rigid-frame bridges are designed to be robust enough to withstand big earthquakes. Since piers are particularly subject to damage from such earthquakes, they have to undergo extra inspections by maintenance workers who watch and check structures carefully after an earthquake has occurred.

Recently, to resist earthquakes persistently, "reinforcement using steel plate", which means rolling steel plate on a pier, has been used on many piers. In such a case, it is difficult to find damage just by looking at the structures.

We have already established knowledge about the relationship between maximum slant and damage to piers during earthquakes. Therefore, if we measure the maximum slant of piers, we can check the damage level of piers 
immediately. This will lead to an increase of efficiency in restoration works and the reduction of the suspension of train operation after an earthquake.

(1) Sensor data collecting with RFID tag

As depicted in figure 10, a slant measuring device is placed on viaducts. A measuring stick is followed by the earthquake response by piers. The jig, which is vertically set at measuring point, mediates between piers and sensors. As a location for the measuring point, we avoid such areas in which damage tends to concentrate. Sensors can measure maximum displacement of both positive and negative directions and record peak values. This sensor, which does not use battery power, shows a high durability and is not so expensive. Pairs of sensors are installed at each place; one is installed in the direction parallel to the rails and the other one is installed at a right angle to the rails. During earthquakes, piers can move in arbitrary directions, so a jig, which can extract displacement of one direction, is attached to the peak sensor and is in touch with the measuring stick. By using this technique, we can measure the maximum displacement of earthquake response in both directions at the same time. The maximum angle data is important for detecting the damage level. Maximum displacement, which is brought about at the measuring point by the inclination of the measuring stick, is measured using the peak sensor. The maximum angle of earthquake response is calculated by using this maximum displacement and length between the fulcrum and the measuring point. The peak sensor is installed at the upper zone of piers of RC rigid frame. In order to obtain sensor data via the RFID tag, which is located in the lower zone of piers, peak sensors and the RFID tag are connected by lead wire (Figure 11). Sensor data is read using the tag reader and the PDA (Figure 2). Figure 12 is a measurement viewer on the PDA. Sensor data consists of the maximum and the minimum value of the $\mathrm{X}$ direction and the $\mathrm{Y}$ direction. Data stored in the PDA are registered on the database server when the maintenance worker gets back to their maintenance office.

(2) Sensor data collecting by radio transmission

By using two radio devices (Figure 4) for damage level detection nodes, the angle data, which is mentioned as "sensor data collecting by using the RFID tag", are transmitted via the relay station (Figure 6) to the database server in the

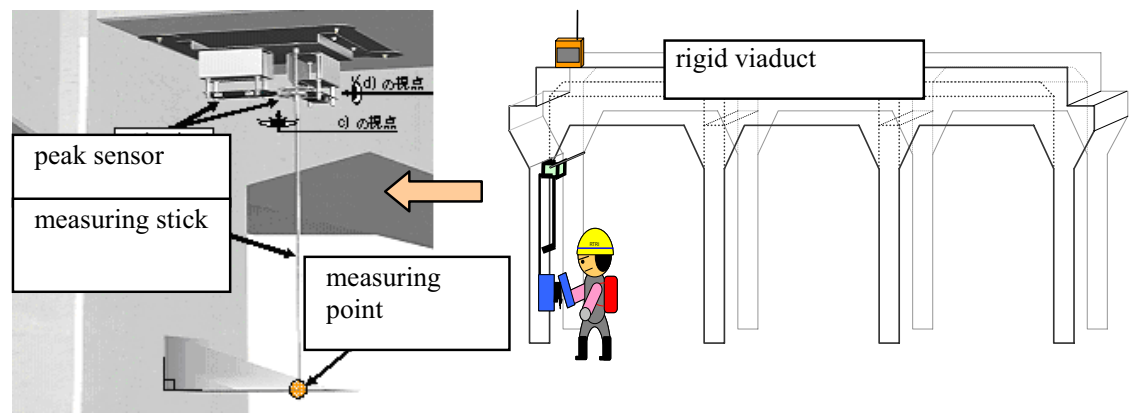

Figure 10: Slant measuring device and its installation. 


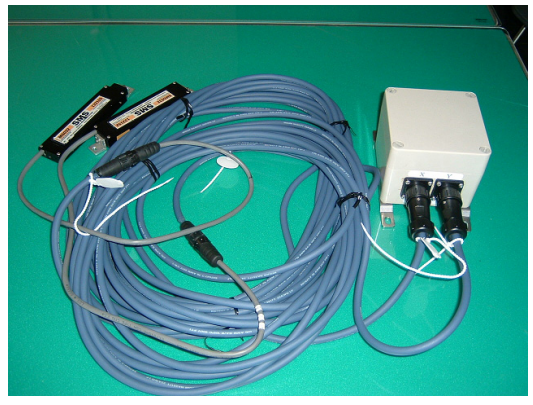

Figure 11: Peak sensor and RFID tag.

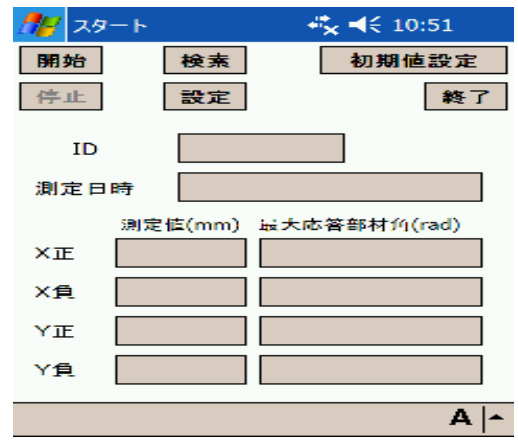

Figure 12: PDA screen for measuring.

administrative area. A data measurement cycle by each node and data transmitting cycle from the relay station to the database can be set arbitrarily.

\subsection{Damage self-detection for the underground part of viaducts}

Foundations of structures are usually constructed under the ground. In the case of bridge piers in running water, their substructure is submerged in water, so it is difficult to visually check the conditions of such structures existing in the ground or in water. Since foundations or substructures of rigid frame bridges, which are a typical structure used in railways, are also in the ground, it is also difficult to visually check the condition of them. For such structures, in order to check whether there is damage caused by earthquakes or other external forces, maintenance workers carefully inspect the underground part by visual checking, and if there is a possibility of deformation in the under ground substructures, nondestructive inspection is applied, or they have to excavate the pier under the ground and visually check them. For safety reasons, there are severe restrictions on the excavation during the operating hours of the train. Therefore they have to excavate in a short time period around midnight when no train is running, which is a very difficult task.

To support the inspection and diagnosis of civil engineering structures, which are difficult to inspect by visual checking, we have developed a self-checking system for underground structures. Figure 13 is an installation image of this system.

As depicted in figure 13, the damage detection sensor is set inside a pier at the point that is subject to damage. A lead line connects this sensor and the RFID tag, which is installed inside the pier above the ground.

Maintenance workers collect sensor data via the RFID tag by radio transmission. We can collect sensor data with a tag reader. This reader is used for RC rigid frames, too. Figure 14 shows the PDA screen when data is being read. The system can also be applied effectively to the inspection of structures in passenger stations. At stations, there are some shops, so piers are covered with ornamental walls. By using this system, we can collect data on the conditions of piers without tearing off the walls. 


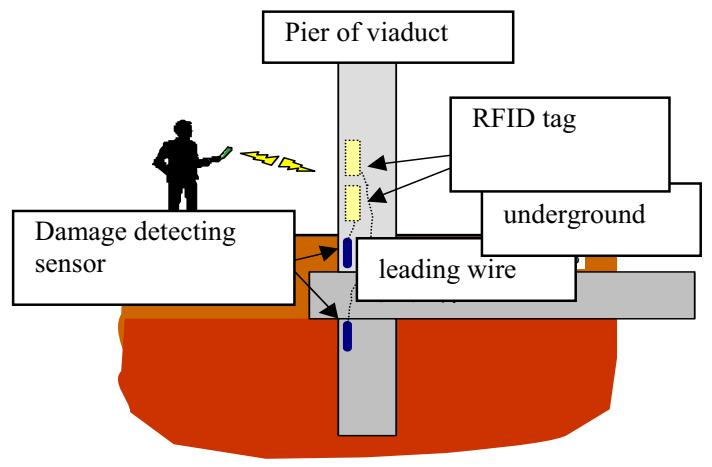

Figure 13: Installation of damage detection sensor.

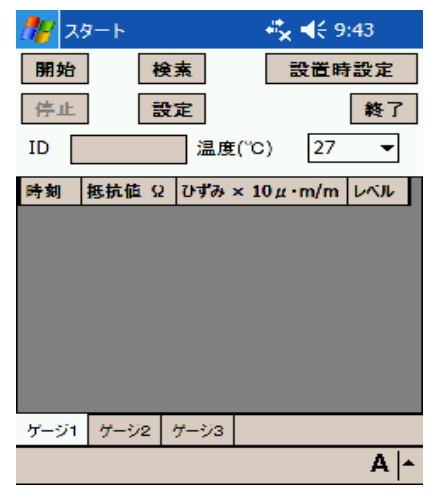

Figure 14: PDA screen for reading data.

\subsection{Detecting cracks in the tunnels}

In order to maintain the safe operation of trains in tunnels, periodic inspections of tunnels, which include visual checks and hammering tests, are carried out. There are several problems in carrying out periodic inspections for wide tunnel linings, such as the lack of maintenance workers, increasing costs of maintenance and the restriction on the closing time of railway tracks. To solve such problems, we have developed a monitoring system for detecting deformation with optical fibers and electro-conductive paint.

These monitoring systems installed up to now have sensors networked with wire. Even if these sensors themselves are simple, such a network can often be an obstacle to the operation of other facilities installed in tunnels. There are also some problems regarding safety and the cost of setting scaffolding and wiring. As a part of these deformation monitoring systems, we examined the applicability of radio transmissions inside a tunnel. We aimed to remove complicated wires and reduce installation costs. Depending on the purpose and combination of sensors, we classify radio transmission systems as follows

(1) Transmission between small-sized sensors

To detect deformation inside a tunnel, $\pi$-shaped sensors are installed at points where there are cracks. In this case, the transmission distance between smallsized sensors need not be long. However, sensors are installed on tunnel linings (especially near the crown part) directly, so data transmission systems attached to sensors need to be small, light and their batteries need to be long life.

(2) Transmission from inside the tunnel to the tunnel entrance

When sensor data are transmitted from inside the entrance to the tunnel via a relay station, this transmission distance needs to be long. In this case, there are some obstacles such as curve sections and stray current.

(3) Transmission from the tunnel entrance to a relay station

Once outside of the tunnel general transmitting techniques (such as wireless LANs) are available. 


\section{Examination on collecting sensor data by using trains}

Collecting sensor data from sensors attached to railway structures via an ad hoc wireless sensor network can be costly. A greater cost is incurred if one sends maintenance workers to the field to collect sensor data manually. If trains, which regularly run on railway lines, can collect sensor data, we will be able to reduce the substantial cost of maintenance work. We carried out an experiment in which we tested whether moving objects, which run at the same speed as a train in service, can receive sensor data properly.

In the test, we set a transmitter of specified low power radio on the ground, which sent one packet message, which contains 6 bytes data every 2 seconds. The data were received by a receiver, which is installed on the back of the driver's seat in a car (Figure 15). Figure 16 shows the relationship between the number of successful message receipts and the driving speed, and Figure 17 shows the relationship between the number of successful message receipts and the transmitting distance.

As is depicted in Figure 16, as the speed of the car increases the number of successful receipts decreases. However, data are certainly received even when the driving speed is $125 \mathrm{~km} / \mathrm{h}$. We suppose that the receivable area depends on the distance. As the speed of the car increases, the connection time between the transmitter on the ground and the receiver installed in the car decreases and the number of successful receipts also decreases. As shown in Figure 17, there is no variety in the number of receiving times even if the transmission distance is varied. The receivable area depends on the directivity of the antenna and the transmission distance between the transmitter and the receiver. If the transmission distance is short, the receivable area would be narrow because the radio has horizontal polarization so the directivity of the antenna is in the longitudinal direction. So we consider that even if the transmitting distance is varied, the receivable area is less varied, there is no variety in the number of receiving times.

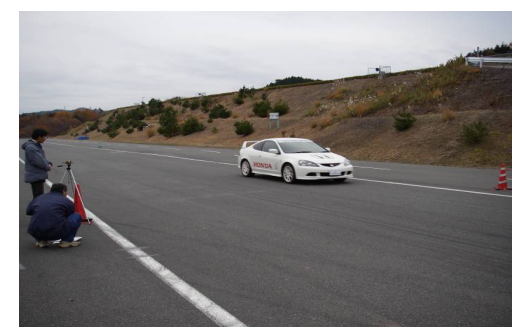

Figure 15: Field test.

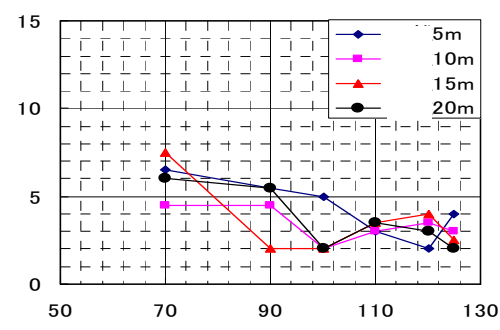

Figure 16: The number of successful receipts (1). 


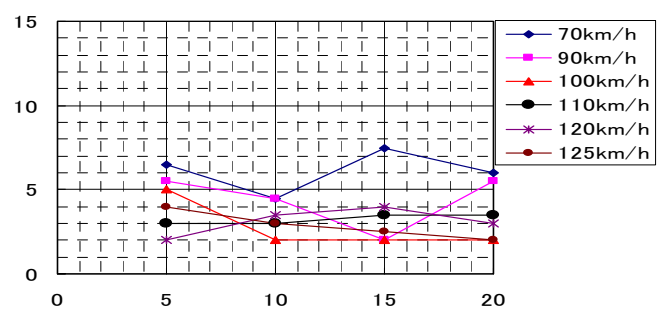

Figure 17: The number of successful receipts (2).

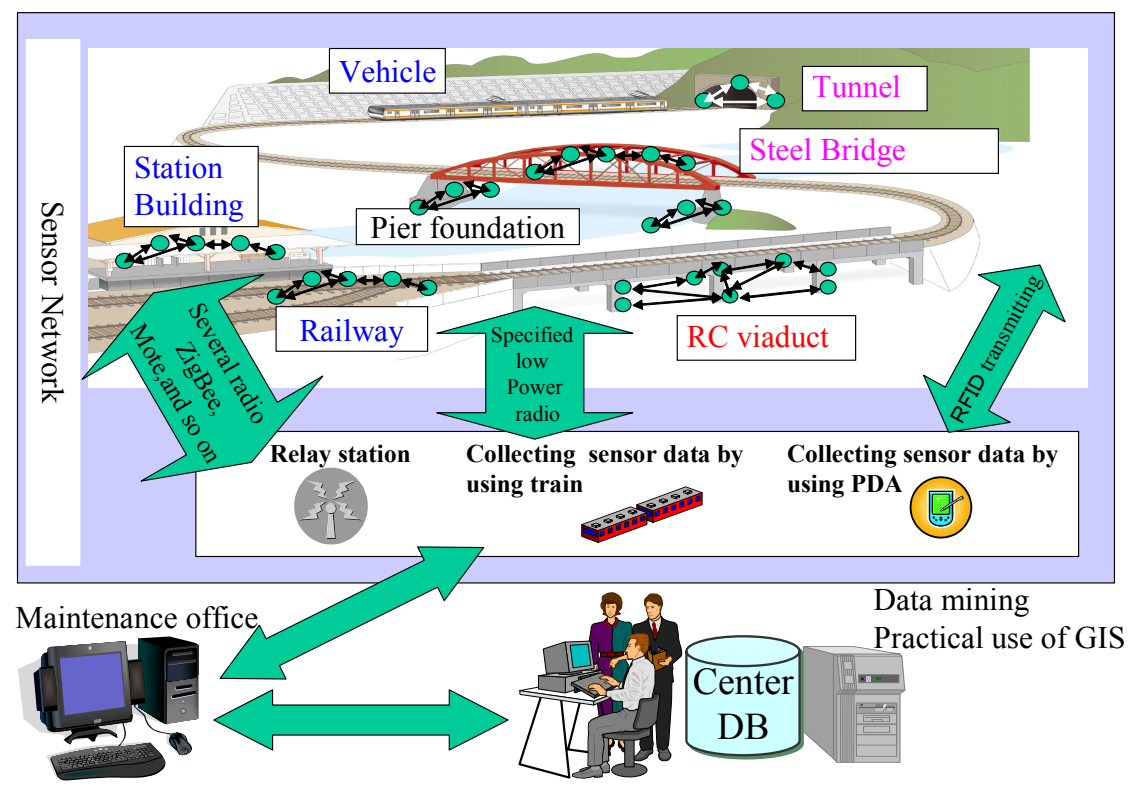

Figure 18: Grand design of the complete system.

We therefore conclude that if the size of data to be sent is sufficiently small, the train, which runs at $125 \mathrm{~km} / \mathrm{h}$, can properly collect sensor data as it runs along the railway lines.

\section{Future works}

We have developed a monitoring system for railway structures by using cuttingedge information technologies. Figure 18 shows a grand design of the complete system that we finally aim at. In fact, there are several issues that we have to 
cope with in order to complete this system. For example, (1) the development of highly reliable sensors that make few errors, (2) the realization of highly waterproof performance and corrosion avoidance (sensors are placed along railway fields where soils contain much water), (3) the determination of threshold values to create an emergency alarm, (4) the development of an efficient and stable power supply system.

We will continue our research to solve these problems and we hope we will be able to put our system to practical use so that it will satisfy the requirements of railway companies, which manage civil-engineering structures.

\section{Acknowledgement}

We carried out this research by using a subsidy from the Ministry of Land, Infrastructure and Transport. 